CERN/TH 98-238

$\mathrm{FNT} / \mathrm{T} 98 / 07$

\title{
Single- and multi-photon final states with missing energy at $e^{+} e^{-}$colliders
}

\author{
G. Montagna ${ }^{a}$, M. Moretti ${ }^{b, c}$, O. Nicrosini ${ }^{a}$ and F. Piccinini ${ }^{a}$ \\ ${ }^{a}$ Dipartimento di Fisica Nucleare e Teorica - Università di Pavia, and \\ INFN - Sezione di Pavia, Via A. Bassi 6, Pavia, Italy \\ ${ }^{b}$ Theory Division, CERN, CH-1211 Geneva 23, Switzerland \\ ${ }^{c}$ Dipartimento di Fisica - Università di Ferrara, and \\ INFN - Sezione di Ferrara, Ferrara, Italy
}

\begin{abstract}
The search for new physics in single- and multi-photon final states with large missing energy at LEP and future $e^{+} e^{-}$colliders requires precise predictions for the Standard Model irreducible background. While at LEP1 the theoretical situation is under control, going to LEP2 (and beyond) some improvements are necessary. To approach the aimed $O(1 \%)$ theoretical accuracy, the tree-level matrix elements for the processes $e^{+} e^{-} \rightarrow \nu \bar{\nu} n \gamma$, with $n=1,2,3$, are exactly computed in the Standard Model, including the possibility of anomalous couplings for single-photon production. Due to the presence of observed photons in the final state, particular attention is paid to the treatment of higher-order QED corrections. Comparisons with existing calculations are shown and commented. An improved version of the event generator NUNUGPV is presented.
\end{abstract}

PACS: $13.10 .+\mathrm{q}, 13.40 . \mathrm{K}, 13.85 . \mathrm{Qk}$

Keywords: electron-positron collisions, new physics searches, irreducible background, visible photons, missing energy, radiative corrections. 


\section{Introduction}

The production of one or more photons and missing energy in high-energy electronpositron $\left(e^{+} e^{-}\right)$collisions is a process of great interest for the scientific programme of LEP and future $e^{+} e^{-}$linear colliders [1, 2].

In the context of precision tests of the electroweak interactions on top of the $Z$ res-

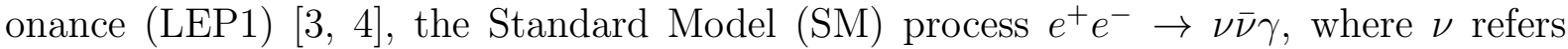
to light neutrinos and $\gamma$ to a detected, energetic photon, has been successfully used for the determination of the number of light neutrino species, in agreement with the result obtained via the measurement of the partial width of the $Z$-boson into invisible particles.

Above the $Z$ resonance, i.e. for current data taking in the LEP2 energy range $(\sqrt{s} \simeq$ $160-200 \mathrm{GeV}$ ) [5] and for planned experiments at the $\mathrm{TeV}$ scale [6], the events with single- and multi-photon final states plus missing energy (E) play an important role in the search for new phenomena beyond the SM [1, 2]. Actually, the SM processes $e^{+} e^{-} \rightarrow \nu \bar{\nu} n \gamma$, with $n=1,2, \ldots$, are the largely dominating irreducible backgrounds to a New Physics (NP) signature consisting of one or more photon(s) and nothing else seen in the detector. Such events can indeed originate from various mechanisms, both in gravity- and gauge-mediated supersymmetric models [7] as well as in scenarios with strong electroweak symmetry breaking [8]. In supersymmetric extensions of the SM, neutralinos, gravitinos and sneutrinos are the lightest supersymmetric particles (LSP) yielding the content of missing energy to the events. Another interesting example of new phenomenon giving rise to $n \gamma+E$ final states is the production of a pair of fourth-generation, heavy neutrinos in association with initial-state radiation (ISR). Finally this signature can be useful to study anomalous triple and quartic gauge couplings. In particular, it can be conveniently used for the study of anomalous couplings as a complementary channel with respect to processes such as $e^{+} e^{-} \rightarrow 4 f, 6 f$, without contamination coming from the vertices involving at least three massive gauge bosons.

The present situation of the theoretical calculations for the above quoted processes can be considered as satisfactory for the purposes of data analysis at LEP1. Going to LEP2, the typical SM cross section is of the order of a few picobarn, yielding thousands of events collected in the four LEP experiments. Hence, there is a demand for theoretical predictions with an accuracy of the order of $1 \%$ for the rate of $e^{+} e^{-} \rightarrow \nu \bar{\nu} n \gamma$ events in the SM. Furthermore, as it will be discussed in the following, the situation concerning the theoretical calculations is not completely satisfactory. In particular, a careful treatment of higher-order QED corrections to processes with detected photons in the final state becomes mandatory for a meaningful comparison between data and theory.

Given the above physics motivations, the aim of the present paper is to perform an exact tree-level calculation in the SM of the $e^{+} e^{-} \rightarrow \nu \bar{\nu} n \gamma$ cross sections, with $n=1,2,3$, supplemented with the most phenomenologically relevant and presently under control radiative corrections. While for one and two photons in the final state exact matrix element calculations have recently appeared in the literature, as it will be discussed in detail in the next Section, no results for three photons are available yet. Concerning ISR, particular care is devoted to the implementation of the effects due to (undetected) 
photon emission. An improved version of the event generator NUNUGPV [9], based on the results here described, is presented for data analysis.

The content of the paper is as follows. In Sect. 2 the status of the available calculations and related programs is critically reviewed, putting special emphasis on the needs of improvement for current experiments at LEP. In Sects. 3 and 4 it is described how some of the existing approximations are overcome in the present study, providing various numerical results. The exact SM calculation of the lowest-order amplitudes for the signatures $e^{+} e^{-} \rightarrow \nu \bar{\nu} n \gamma$, with $n=1,2,3$, is discussed in Sect. 3, while Sect. 4 is devoted to the implementation of higher-order QED radiative corrections. In passing, numerical results for integrated cross sections as well as comparisons with existing calculations are shown and commented. The impact of the theoretical improvements on exclusive photon distributions is discussed and compared with typical NP effects in Sect. 5. The main conclusions and open issues are drawn in Sect. 6.

\section{Status of the theoretical predictions and generators}

Concerning the process $e^{+} e^{-} \rightarrow \nu \bar{\nu} \gamma$, described by the Feynman diagrams depicted in Fig. 1, several calculations, with a different degree of accuracy, are known in the literature.

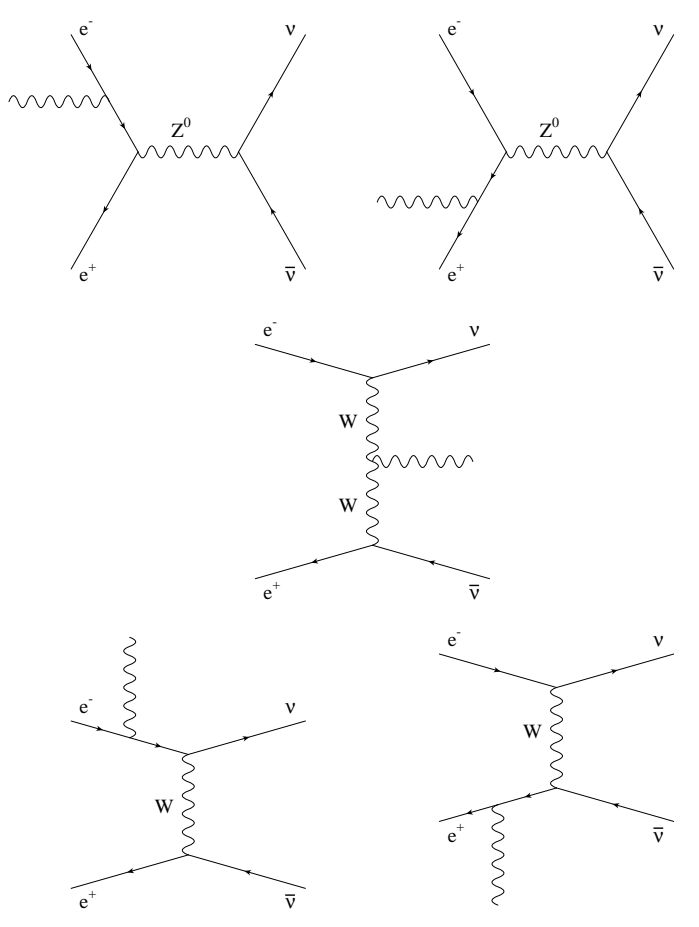

Figure 1: The tree-level Feynman diagrams for the process $e^{+} e^{-} \rightarrow \nu \bar{\nu} \gamma$.

Before the start of LEP/SLC operations, the status of the theoretical calculations and 
related computational tools has been summarized in Ref. 10 and, more recently, in view of the energy upgrade of the LEP collider from LEP1 to LEP2 regime, in Ref. [11].

First attempts [12] to compute the cross section associated to the graphs of Fig. 1] consider the limit $M_{W} \rightarrow \infty$ and neglect the contribution of the non-abelian $W W \gamma$ vertex (the so-called Point Interaction Approximation). In other approximate calculations the invisible neutrino-pair cross section is dressed with some (universal) radiation factor to attach one external photon to the charged fermion legs, e.g. by using an angular dependent radiator [13, 14, a parton shower (PS) algorithm (as in the program PYTHIA) [15] or the Yennie-Frautschi-Suura (YFS) exclusive exponentiation (as done in KORALZ) [16]. By construction, these approaches allow to account for the leading contributions due to the collinear and infrared singularities but need to be corrected for the effect of sub-leading terms and/or internal photon radiation from the off-shell $W$ boson (as recently done in an approximate way in Ref. [17]) that are contained in the exact matrix element. The first complete calculation of the matrix element of the process $e^{+} e^{-} \rightarrow \nu \bar{\nu} \gamma$ was done in Ref. [18]. The corresponding exact matrix element is implemented in the event generator MMM [19]. In Ref. [18], by working in the approximation of neglecting in the squared matrix element terms with at least three boson propagators, also a compact, analytical expression for the differential spectrum on the energy and angle of the observed photon is obtained, yielding the result

$$
\frac{d \sigma}{d \cos \vartheta_{\gamma} d k}=\frac{\alpha}{12 \pi^{2}} G_{F}^{2} M_{W}^{4} \frac{s^{\prime} k}{s k_{+} k_{-}}\left[\eta_{+}^{2} F\left(\eta_{+}\right)+\eta_{-}^{2} F\left(\eta_{-}\right)\right] .
$$

The meaning and explicit expression of the symbols entering eq. (1) can be found in Ref. [18]. The photon spectrum of eq. (1) contains the bulk of the contributions due to $W$-boson exchange and agrees within $1 \%$ with approximate calculations discussed above for center of mass (c.m.) energies around the $Z$ resonance [14]. The analytical photon spectrum of eq. (11) is implemented in the event generator NUNUGPV [9].

Concerning radiative corrections, the exact one-loop electroweak corrections to $e^{+} e^{-} \rightarrow$ $\nu \bar{\nu} \gamma$ process are not yet available. What is presently known is the full set of one-loop electroweak corrections to the "sub-process" $e^{+} e^{-} \rightarrow Z \gamma$, with an on-shell final $Z$ boson [20], and the subset of one-loop QED corrections to the $Z$-exchange contribution to $\nu \bar{\nu} \gamma$ final state [18, 21]. However, since, contrary to LEP1, the $W$-boson contributions are essential at LEP2, as clearly shown in Fig. \& for different selection criteria, the still missing part of the full one-loop calculation due to the corrections to the dominant diagrams with $W$-boson exchange is necessary in order to reach the aimed theoretical precision at the $1 \%$ level. Actually, one can see from Fig. 2 that the relative contribution of $W$-diagrams (squared modulus and interference terms) can amount to about $60 \%(80 \%)$ of the full cross section, including or excluding, via a cut on the missing mass of the event, the $Z$ return. Therefore, in the absence of a full $O(\alpha)$ electroweak calculation, the goal of a $1 \%$ theoretical accuracy in the predictions for single-photon plus missing energy production turns out to be difficultly reachable at present. However, in order to take care of the most sizeable higher-order corrections, the lowest-order calculations are typically improved by the inclusion of the (large) effects due to ISR. In mostly used computational tools such a 

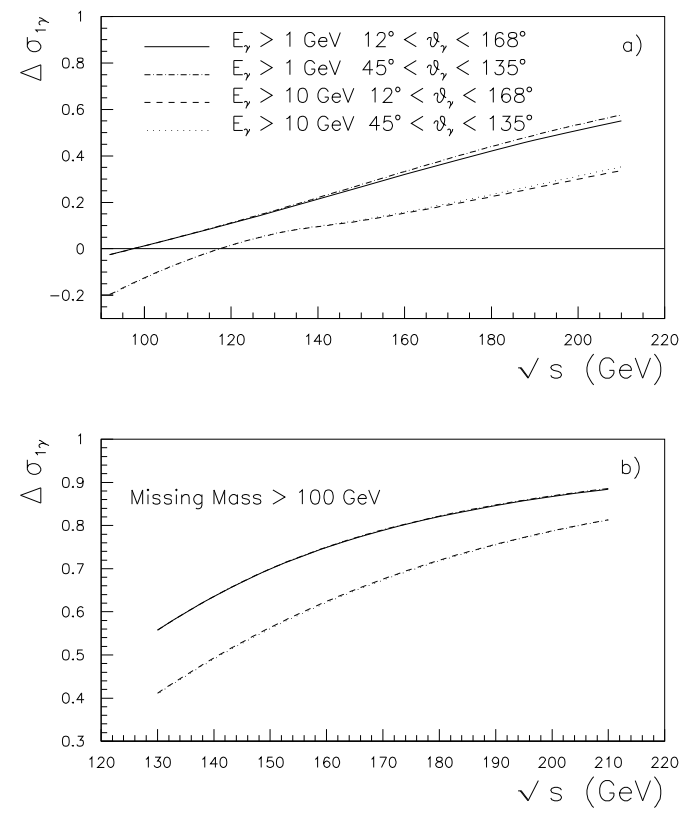

Figure 2: The relative size of the $W$-exchange diagrams with respect to the total cross section of the signature $\nu \bar{\nu} \gamma$, for different experimental cuts. The quantity $\Delta \sigma_{1 \gamma}$ is defined as: $\Delta \sigma_{1 \gamma}=1-3 \sigma\left(\nu_{\mu} \bar{\nu}_{\mu} \gamma\right) / \sigma(\nu \bar{\nu} \gamma)$. In Fig. $2 \mathrm{a}$ the four lines correspond to the cuts on the photon energy and angle specified in the plot; Fig. 2b is the same as Fig. 2a, with an additional cut on the missing mass of the event.

contribution is taken into account via traditional algorithms for computing QED radiative corrections in the leading logarithmic (LL) approximation, such as the PS algorithm 22] (as in PYTHIA), the Structure Function (SF) approach 223 (as in MMM and NUNUGPV, but also in PYTHIA) and YFS exclusive exponentiation 24 (as in KORALZ). Two different variations of the SF method are employed in MMM and NUNUGPV. SFs in strictly collinear approximation are used in MMM, while in NUNUGPV $p_{t}$-dependent SFs [13, 25] are implemented to improve the treatment of ISR with the effect of the transverse degrees of freedom at the LL level [9] (this point will be further discussed later).

The previously quoted programs KORALZ, MMM and NUNUGPV are the standard Monte Carlo generators used by the LEP collaborations for the analysis of the data relative to the events $e^{+} e^{-} \rightarrow \nu \bar{\nu} \gamma(\gamma)$ [2]. As already emphasized, these programs differ both in the treatment of the lowest-order matrix element and in the implementation of higher-order corrections. Actually, the agreement between the above generators, whenever compared to estimate the theoretical precision against the statical accuracy in the measured cross section (of the order of a few per cent), is fairly good for exclusive single-photon final states (say for photon energies above approximatively $10 \mathrm{GeV}$ ), whereas for low photon 
energies and more inclusive final states the agreement is not at present satisfactory [26]. Therefore, since in the search for new stable, neutral particles one is interested in detecting an excess of events at low photon energies [26], the present status points out the need of improving theoretical predictions to avoid a loss of sensitivity to NP searches in radiative events at LEP2.

Let us come now to discuss the present status of the theoretical predictions for the process $e^{+} e^{-} \rightarrow \nu \bar{\nu} \gamma \gamma$, which is the most relevant SM background to a signature with two acoplanar photons and large missing energy. Only very recently, dedicated calculations appeared in the literature, as motivated by the search of anomalous $\gamma \gamma+E$ events at LEP. A complete diagrammatic calculation, using the helicity amplitude technique, supplemented with collinear SFs to account for ISR, was done in Ref. [27]. This paper confirmed a previous evaluation in Born approximation contained in Ref. [28]. Approximate predictions for the process of interest are obtained by the LEP collaborations by using the above quoted programs with QED "dressing" of the neutrino-pair cross section, namely PYTHIA (via the PS) and KORALZ (via the YFS method). Further, modern packages for the automatic calculation of Feynman amplitudes, such as GRACE [29] and CompHEP [30, are used by the experiments to calculate the cross section and generate events. Both packages implements collinear SFs for ISR, with an option for PS in GRACE.

Quite recently, an extensive comparison, at the level of total and differential cross sections, between all the available calculations has been performed in Ref. [31]. This detailed comparison shows a (dis)agreement between independent calculations at 10-20\% level (or worse) and, more generally, an unsatisfactory situation about the software for the analysis of the data with acoplanar photons (see also last paper in Ref. [2]).

\section{Calculation of the lowest-order cross sections}

In order to approach the $O(1 \%)$ theoretical precision and improve the predictions of the earlier version of the program NUNUGPV, the lowest-order matrix elements associated to the processes $e^{+} e^{-} \rightarrow \nu \bar{\nu} n \gamma$ (with $n=1,2,3$ ) have been exactly calculated in the SM.

The matrix element for single-photon production has been computed by means of helicity amplitude techniques [32], including the possibility of anomalous $\Delta k_{\gamma}$ and $\lambda_{\gamma}$ contributions to the $W W \gamma$ coupling. This offers the possibility of exploiting the LEP statistics relative to $\gamma+E$ events in order to put constraints on such anomalous terms. The diagrammatic calculation has been cross-checked by using the algorithm ALPHA 33] and found to be in perfect agreement. The three-body phase space has been generated recursively, by decomposing the phase-space element $d \Phi_{3}$ as follows

$$
d \Phi_{3}\left(P \rightarrow k, q_{1}, q_{2}\right)=d \Phi_{2}(P \rightarrow k, Q)(2 \pi)^{3} d Q^{2} d \Phi_{2}\left(Q \rightarrow q_{1}, q_{2}\right),
$$

where $P=p_{1}+p_{2}$ is the total incoming momentum, $Q=q_{1}+q_{2}$ is the total momentum carried by the neutrinos and $k$ is the photon momentum. The independent kinematical

variables are chosen to be: $E_{\gamma}, \cos \vartheta_{\gamma}, \phi_{\gamma}, \cos \theta_{\nu}^{*}, \phi_{\nu}^{*}$, where the photon variables are 

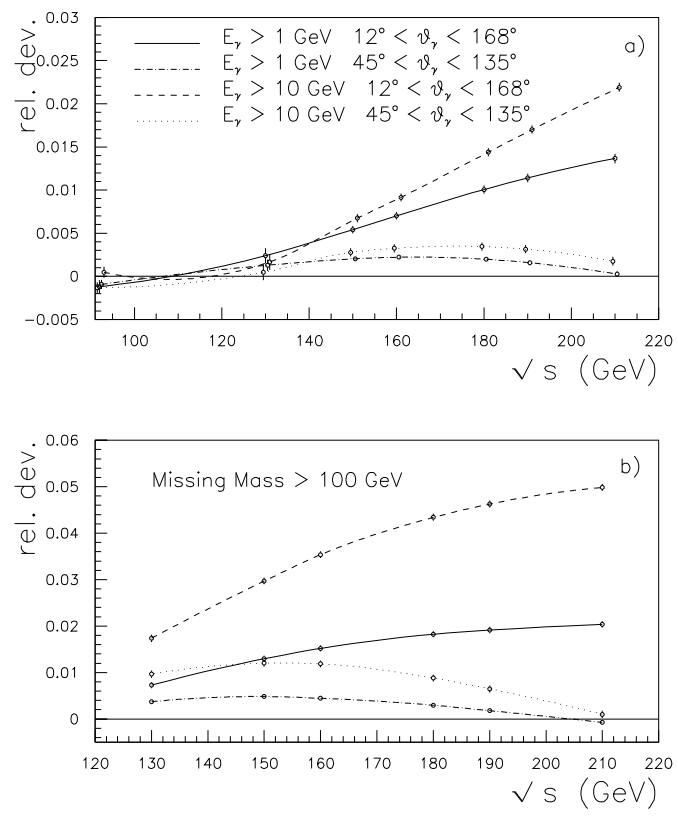

Figure 3: The relative deviation between the exact $e^{+} e^{-} \rightarrow \nu \bar{\nu} \gamma$ cross section and the approximated one, as obtained via eq. (1) implemented in the earlier version of NUNUGPV. The differences are shown for the event selections given in Fig. 3a, with an additional cut on the missing mass in Fig. $3 \mathrm{~b}$.

generated in the c.m. frame, while the neutrino ones in the reference frame where $\overrightarrow{q_{1}}+\overrightarrow{q_{2}}=$ 0 . Since the photon energy can be expressed in terms of the invariant mass $Q^{2}$ via the linear relation $E_{\gamma}=\left(s-Q^{2}\right) / 2 \sqrt{s}$, where $s$ is the total c.m. energy, it is convenient to generate $Q^{2}$ in such a way to sample directly the leading matrix element configurations, that are due to the emission of a soft photon or a hard, $Z$ return one. This importance sampling strategy is followed in the Monte Carlo integration in order to cure the variance of the matrix element in correspondence of the infrared and $Z$ return peaking behaviour. The photon angle is generated according to the weight function $p\left(\cos \vartheta_{\gamma}\right) \propto 1 /\left(1-\beta^{2} \cos ^{2} \vartheta_{\gamma}\right)$, with $\beta=\sqrt{1-4 m_{e}^{2} / s}$, to take care of the collinear peaking.

The exact treatment of the single-photon matrix element upgrades the released version of NUNUGPV, based on the photon spectrum of eq. (11) of Ref. [18], to include previously neglected $W$-boson effects relative to contributions with at least three boson propagators. The size of such previously neglected effects is shown in Fig. 3, for typical event selections used by the LEP experiments [2, 26]. The calculation of the single-photon cross section obtained with the exact matrix element is compared with the cross section resulting from the integration of the photon spectrum of eq. (1). The relative difference between the two calculations is at a few per cent level, both without and with a cut on the missing mass, 
in agreement with the degree of approximation stated in Ref. 18. However, it should be noticed that an exact treatment of the lowest-order matrix element is actually mandatory at LEP2 if a theoretical accuracy of the order of $1 \%$ is aimed at.
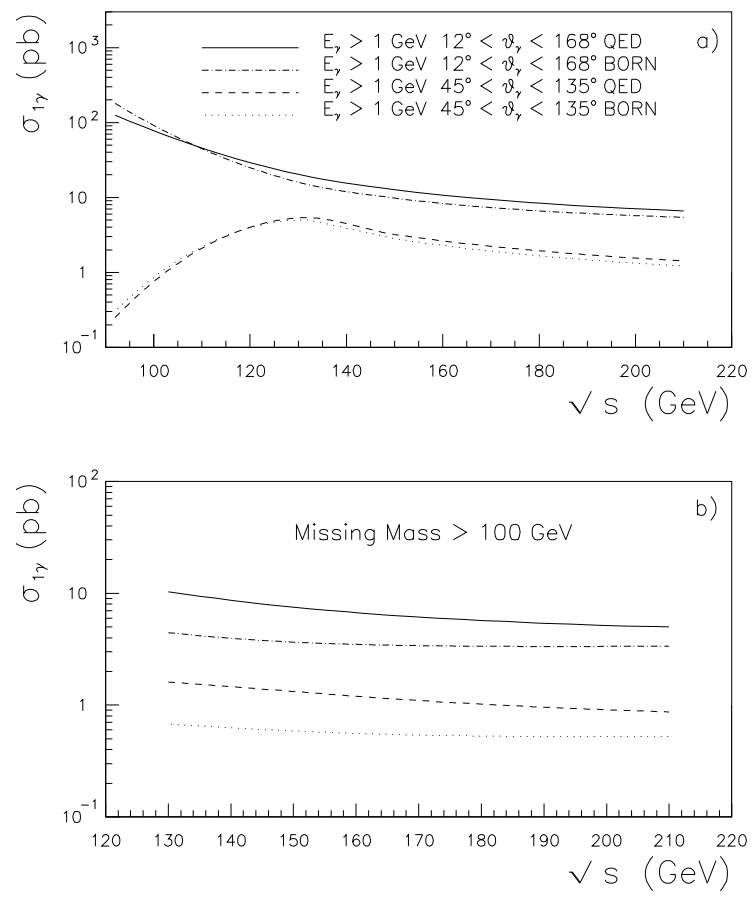

Figure 4: The tree-level cross section for the process $e^{+} e^{-} \rightarrow \nu \bar{\nu} \gamma$ as compared with the cross section with higher-order QED corrections, obtained by using collinear SFs. Two typical selection criteria, specified in Fig. 4a, are considered, including (Fig. 4a) and excluding, via a cut on the missing mass (Fig. 4b), the $Z$ radiative return.

The already quoted algorithm ALPHA, that is conceived for the automatic computation of tree-level multi-particle production amplitudes without any need of Feynman graphs expansion, has been employed for the calculation of the matrix elements with two and three photons in the final state. For the case $\nu \bar{\nu} \gamma \gamma \gamma$ the calculation here presented is the first one appearing in the literature. Without entering the details of the algorithm ALPHA, which is fully discussed in the literature [33], it is worth noticing, for the aim of the present study, that the predictions of this automatic algorithm have been already compared with the diagrammatic results for the processes $e^{+} e^{-} \rightarrow 4 f$ [11, 34, showing excellent agreement, and also successfully used to obtain original results for other reactions such as $\gamma \gamma \rightarrow 4 f$ [35], confirmed in Ref. [36], $e^{+} e^{-} \rightarrow 4 f+\gamma$ [37] and $e^{+} e^{-} \rightarrow 6 f$ [38]. Concerning the treatment of phase space, the decomposition introduced for two photons 
in the final state reads as follows

$$
d \Phi_{4}\left(P \rightarrow k_{1}, k_{2}, q_{1}, q_{2}\right)=d \Phi_{3}\left(P \rightarrow k_{1}, k_{2}, Q\right)(2 \pi)^{3} d Q^{2} d \Phi_{2}\left(Q \rightarrow q_{1}, q_{2}\right),
$$

where, as before, $Q=q_{1}+q_{2}$ is the total momentum carried by the neutrinos and $k_{1}, k_{2}$ are the momenta of the two photons. The independent kinematical variables are chosen to be: $Q^{2}, E_{\gamma, 1}, E_{\gamma, 2}, \cos \vartheta_{\gamma, 1}, \phi_{\gamma, 1}, \phi_{\gamma, 12}, \cos \theta_{\nu}^{*}, \phi_{\nu}^{*}$, where the photon variables are generated in the c.m. frame, while the neutrino ones in the reference frame where $\overrightarrow{q_{1}}+\overrightarrow{q_{2}}=0$. As in the case of single-photon production, the energies of the photons are generated according to the soft and $Z$ return peaking structure, whereas one of the photon angles follow the collinear behaviour. A generalization of the above strategy is also followed for three photons in the final state.

Some numerical results for the lowest-order cross sections of the processes $e^{+} e^{-} \rightarrow \nu \bar{\nu} \gamma$ and $e^{+} e^{-} \rightarrow \nu \bar{\nu} \gamma \gamma$ are shown, as functions of the c.m. energy in the LEP2 energy range, in Fig. 1 and Fig. . The input parameters used throughout the present study are the same as in Ref. 14]. By comparing Fig. 国and Fig. 5, it can be seen that the cross section
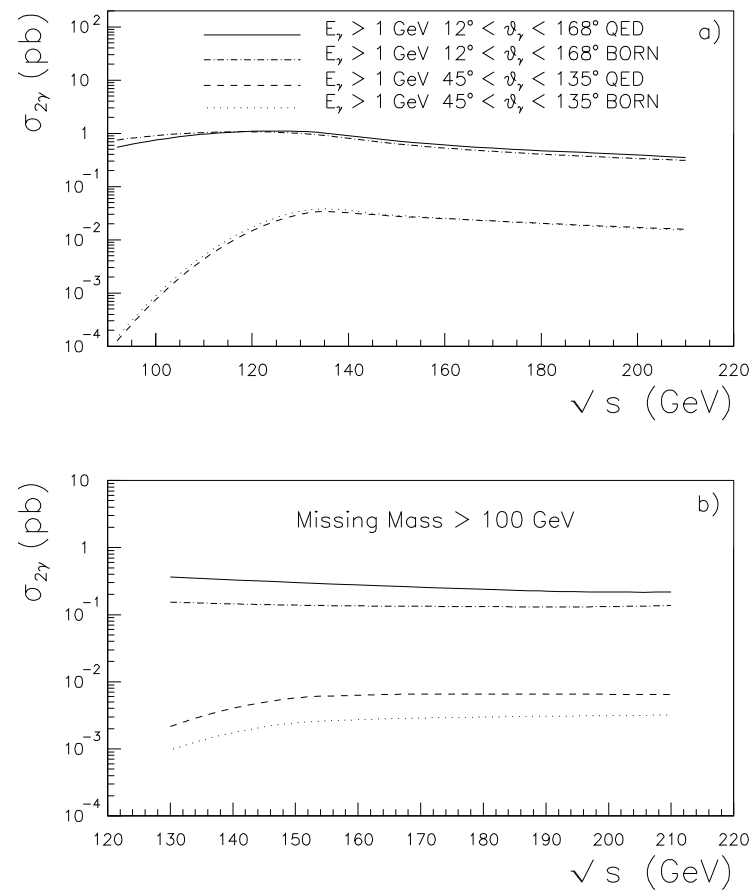

Figure 5: The same as Fig. 4 for the process $e^{+} e^{-} \rightarrow \nu \bar{\nu} \gamma \gamma$.

for the signature $\nu \bar{\nu} \gamma \gamma$ is about a factor 10-100 smaller than the cross section for $\nu \bar{\nu} \gamma$, the reduction factor being strongly dependent, as expected, on the imposed photon cuts. A similar ratio is present in the LEP2 range for the $\nu \bar{\nu} \gamma \gamma \gamma$ cross section with respect to the one for the $\nu \bar{\nu} \gamma \gamma$ final state. 


\section{Treatment of higher-order QED corrections}

Because the level of accuracy demanded to the calculations for single- and multi-photon production with missing energy should reach the $O(1 \%)$ precision, the most important radiative corrections must be necessarily included. To this end, all the calculations known in the literature take into account the effect of ISR. As already discussed in Sect. 2, this goal is achieved by using standard algorithms for universal photonic corrections, such as the PS method, the YFS exponentiation and the SF approach. In particular, the SF approach, because of its simplicity, is certainly the most widely used algorithm, implemented in many generators of interest here, such as CompHEP, GRACE, MMM and NUNUGPV. More precisely, as discussed in Sect. 2, all the programs make use of SFs in strictly collinear approximation, while in NUNUGPV $p_{t}$-dependent SFs [13, 25] are implemented to improve the treatment of ISR by including $p_{t} / p_{L}$ effects. Because of the presence of photons among the observed final-state products, the inclusion of the ISR requires a particular care. This caution is further motivated by the very large enhancement of the lowest-order cross sections as due to the ISR and clearly visible in Fig. 14 and Fig. 5. As can be seen, the single- and double-photon cross section are significantly enhanced when considering typical event selections. The enhancement factor is about 1.3 when including the $Z$ return and about 2 when excluding it. As done in many practical applications [14, 19, 27], the above results can be simply obtained by convoluting the hard-scattering cross section of interest with collinear SFs, according to the factorized formula

$$
\sigma_{\text {coll }}=\int d x_{1} d x_{2} D\left(x_{1}, s\right) D\left(x_{2}, s\right) d \sigma \Theta(\text { cuts }) .
$$

It allows to take into account the impact of higher-order QED corrections, due to photon emission before the hard-scattering reaction (pre-emission), at the LL level. It has been checked that the dominant effects of the ISR obtained according to eq. (4) numerically agree, for different experimental set up, with those already known in the literature for one and two photons in the final state [14, 27, 31]. Equation (14) is a good approximation to QED radiative corrections in the LEP1 energy regime at the LL level, since, with the standard selection criteria, hard "pre-emission" photons imply the production of $\nu \bar{\nu}$ pairs off $Z$-resonance and are therefore inhibited. On the contrary, going to LEP2 it can be easily realized that the implementation of the ISR as given by eq. (乐) is an approximation that clearly fails whenever the photonic degrees of freedom, of the pre-emission and hardscattering process, respectively, overlap in the same phase space region. Actually, because the collinear SFs can be seen as the result of an integration over the angular variables of the photon radiation, eq. (4) does not take into account the correct statistical factor to be included for the presence of identical particles in the final state. Furthermore, if the pre-emission photon is detectable, the reconstruction of the event via eq. (雨) is only approximate and this might imply an additional inaccuracy. Therefore, one should expect that the implementation of the ISR as given by eq. (41) leads to an overestimate of the higher-order QED corrections. This effect is clearly dependent on the photon(s) detection criteria and can be expected to be not negligible with respect to an $O(1 \%)$ theoretical accuracy. An estimate of the effects due to the phase space overlapping of the IS pre- 
emission photons with the observed ones can be obtained by supplying the QED SFs with the transverse degrees of freedom. Actually, the generation of the angular variables at the level of the ISR gives the possibility of rejecting in the event sample those pre-emission photons above the minimum detection angle, thus avoiding "overlapping effects".

According to such a procedure, the cross section with higher-order QED corrections can be calculated as follows (for the realistic data sample of at least one photon)

$$
\begin{aligned}
\sigma^{1 \gamma(\gamma)}=\int d x_{1} d x_{2} d c_{\gamma}^{(1)} d c_{\gamma}^{(2)} & \tilde{D}\left(x_{1}, c_{\gamma}^{(1)} ; s\right) \tilde{D}\left(x_{2}, c_{\gamma}^{(2)} ; s\right) \Theta(\text { cuts }) \\
\times & \left(d \sigma^{1 \gamma}+d \sigma^{2 \gamma}+d \sigma^{3 \gamma}+\ldots\right)
\end{aligned}
$$

where $\tilde{D}\left(x, c_{\gamma} ; s\right)$ [9] is a proper combination of the collinear SF $D(x, s)$ with an angular factor inspired by the leading behaviour $1 /(p \cdot k)$. The latter is introduced to generate the angular variables of the pre-emission photons. According to eq. (5), an "equivalent" photon is generated for each colliding lepton and accepted as a higher-order ISR contribution if:

- the energy of the equivalent photon is below the threshold for the observed photon $E_{\gamma, \min }$, for arbitrary angles; or

- the angle of the equivalent photon is outside the angular acceptance for the observed photons, for arbitrary energies.

Within the angular acceptance of the seen photon(s), the cross section is evaluated by summing the exact matrix elements for the processes $e^{+} e^{-} \rightarrow \nu \bar{\nu} n \gamma, n=1,2,3$ $\left(d \sigma^{1 \gamma}, d \sigma^{2 \gamma}, d \sigma^{3 \gamma}\right)$. Notice that from the point of view of computing $\sigma^{1 \gamma(\gamma)}$ the real contributions $d \sigma^{n \gamma}, n \geq 2$, represent the "hard" radiative corrections to be matched with the universal soft+virtual ones accounted for by the SFs. Therefore they are in principle necessary at all orders. The truncation of hard radiative corrections at the level of $d \sigma^{3 \gamma}$ introduces a spurious infra-red sensitivity in radiative corrections at the order $\alpha^{4} \ln ^{4}\left(E / E_{\gamma, \min }\right)$ which, from the practical point of view, is completely negligible at realistic $E_{\gamma, \min }$. Since the radiative corrections implemented by means of this procedure are at the LL level, its theoretical error is dominated by missing truly $O(\alpha)$ corrections.

In order to quantify the overestimate introduced by the collinear SFs in the calculation of the ISR via eq. (4), the relative difference between eq. (4) and eq. (5) is shown in Fig. 6, for several photon detection criteria. As can be seen, at LEP1 the overlapping effects are contained within a few per mille, and therefore negligible on the scale of the experimental accuracy. Going to LEP2 energies, the impact of "overlapping effects" I varies within 1-4\% when including the $Z$ return and is still larger, reaching $10 \%$, when imposing a cut on the missing mass, as usually done in realistic event selections. This effect is therefore important in the light of the aimed theoretical precision. It should be noticed, however,

\footnotetext{
${ }^{1}$ Notice that, as already remarked, by "overlapping effects" two effects are understood, which do occur simultaneously whenever two or more photons are detected: in eq. (4) $i$ ) the statystical factor is incorrectly accounted for, $i i$ ) the reconstruction of the event is approximate.
} 

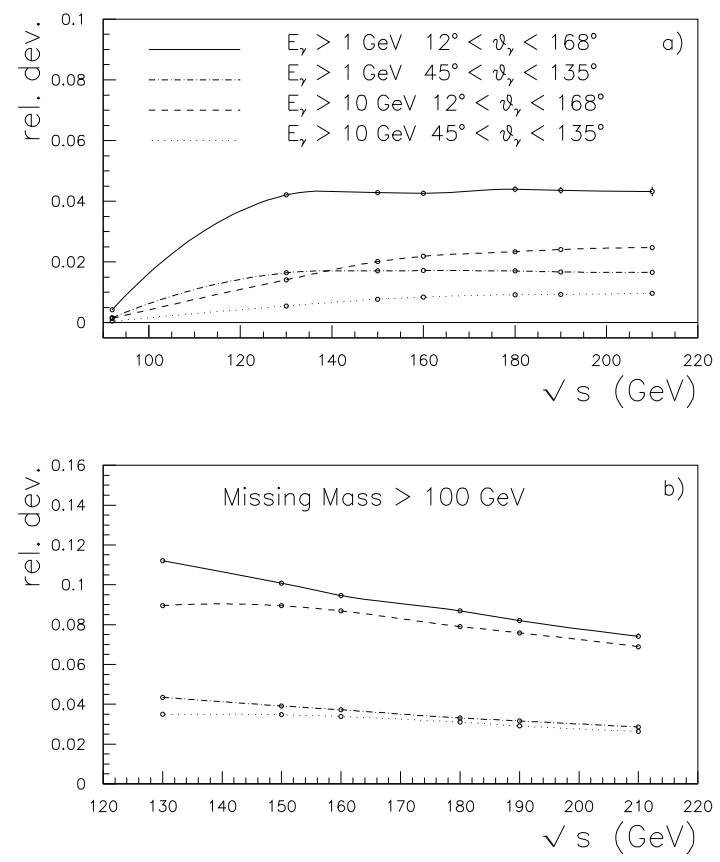

Figure 6: Contribution of "overlapping effects" (see the text for definition) to the cross section for the process $e^{+} e^{-} \rightarrow \nu \bar{\nu} \gamma(\gamma)$. In Fig. 2a the four lines correspond to the cuts on the photon energy and angle specified in the plot; Fig. $2 \mathrm{~b}$ is the same as Fig. 2a, with an additional cut on the missing mass of the event.

that, where the difference reaches a ten per cent size, the overall effect of ISR is to enhance the tree-level cross section of a factor of two. A qualitative explanation of the overlapping effects discussed above can be given as follows. The overestimate of radiative corrections takes place when the pre-emission photon can reach the observability region for the detected photon. At LEP1, and with standard selection criteria, the emission of multiple detectable photons implies that neutrino production occurs off $Z$-resonance and therefore the overlapping effect is naturally suppressed by the dynamics. At LEP2, where this suppression is no longer active, the overlapping effects can become more sizeable, depending on the angular acceptance and minimum energy of the observed photons, and, more generally, in the presence of additional cuts on the four-momenta of the observed photons (as in the case of a cut on the missing mass).

Analogously to eq. (5), the QED corrected cross section for the signature of at least two-photons in the final state can be cast as follows

$$
\begin{aligned}
& \sigma^{2 \gamma(\gamma)}=\int d x_{1} d x_{2} d c_{\gamma}^{(1)} d c_{\gamma}^{(2)} \tilde{D}\left(x_{1}, c_{\gamma}^{(1)} ; s\right) \tilde{D}\left(x_{2}, c_{\gamma}^{(2)} ; s\right) \Theta(\text { cuts }) \\
& \times\left(d \sigma^{2 \gamma}+d \sigma^{3 \gamma}+\ldots\right)
\end{aligned}
$$


It is worth noticing that $d \sigma^{3 \gamma}$ in eq. (6) plays the same role as $d \sigma^{2 \gamma}$ in eq. (5), and hence is a key ingredient when considering the signature with at least two photons in the final state. Numerical results for such a signature are given in Fig. 7, showing the relative difference between eq. (4) and eq. (6). Also for the process $e^{+} e^{-} \rightarrow \nu \bar{\nu} \gamma \gamma(\gamma)$ the implementation of the ISR via collinear SFs can lead to an overestimate of the physical cross section of the order of several per cent.
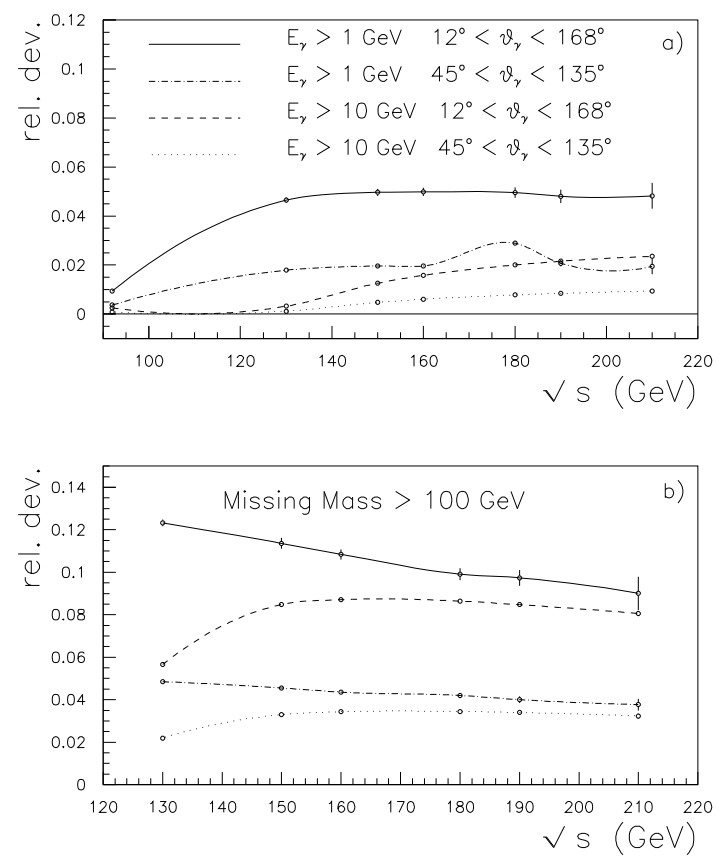

Figure 7: The same as Fig. 6 for the process $e^{+} e^{-} \rightarrow \nu \bar{\nu} \gamma \gamma(\gamma)$.

\section{Distributions}

The results shown in the previous sections refer to integrated cross sections. However, in the search for NP effects, one is also interested in more exclusive distributions involving the photon(s) energy and angle. Therefore, it is worth studying how the theoretical improvements discussed above on tree-level matrix elements and higher-order QED corrections have an impact on photon distributions with respect to typical NP deviations.

To this aim a few results obtained by using the improved version of NUNUGPV as an event generator are illustrated in Figs. $8-10$. Figure 8 and Fig. 9 refer to the signature $e^{+} e^{-} \rightarrow \nu \bar{\nu} \gamma(\gamma)$, while Fig. 9 is relative to the process with at least two photons, i.e. 

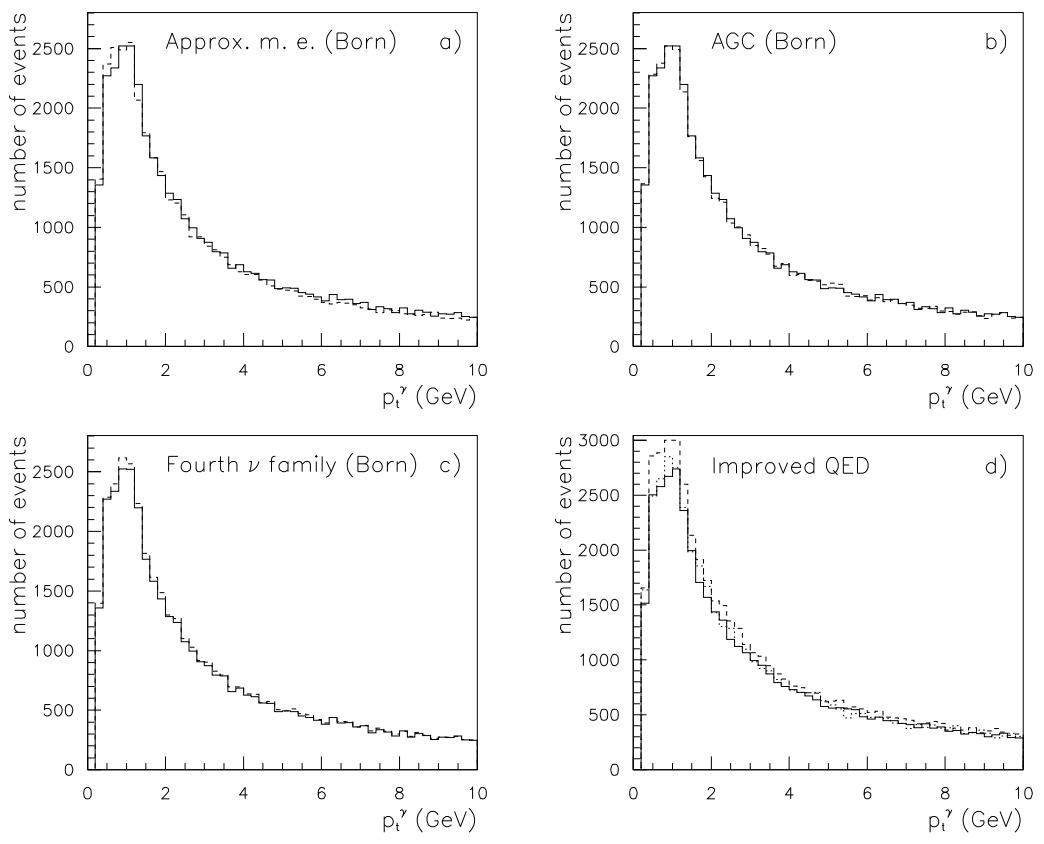

Figure 8: "Soft" $p_{t}^{\gamma}$ distribution for the leading photon of the process $e^{+} e^{-} \rightarrow \nu \bar{\nu} \gamma(\gamma)$ at $\sqrt{s}=190 \mathrm{GeV}$. The cuts on the observed photons are $E_{\gamma}>1 \mathrm{GeV}$ and $12^{\circ}<\vartheta_{\gamma}<168^{\circ}$. Exact tree-level calculation (solid line) versus: approximate analytic spectrum of Ref. [18] (dashed line) (Fig. 8a), exact SM calculation plus anomalous couplings contribution (dashed line) (Fig. 8b), exact SM calculation plus massive $\left(m_{\nu}=50 \mathrm{GeV}\right)$ fourth family neutrinos (dashed line) (Fig. 8c). In Fig. 8d ISR correction is implemented according to: eq. (5) (solid line), eq. (四) (dashed line) and via SFs with $p_{t} / p_{L}$ effects as in the released version of NUNUGPV (dotted line).

$e^{+} e^{-} \rightarrow \nu \bar{\nu} \gamma \gamma(\gamma)$. For the sake of comparison, the histograms shown in Figs. $8-10$ are normalized to the same luminosity. A typical LEP2 energy of $\sqrt{s}=190 \mathrm{GeV}$ is considered, with the cuts $E_{\gamma}>1 \mathrm{GeV}$ and $12^{\circ}<\vartheta_{\gamma}<168^{\circ}$ for the observed photons.

As an example, the $p_{t}^{\gamma}$ distribution of the most energetic photon (leading photon) is shown in Fig. \& for the "soft" region and in Fig. 98 for the "hard" one. The first three plots (Figs. 8a-8c, Figs. 9a-9c) do not take into account the contribution of ISR (Born approximation), while the last one (Fig. 8d, Fig. 9d) shows QED corrected distributions according to different realizations. The aim is to show how typical NP effects may compete with an improved calculation of the SM background, both at the level of tree-level matrix elements and higher-order QED corrections, previously discussed. Actually, in the first three plots the solid line is the lowest-order prediction obtained by means of the exact single-photon matrix element as compared with the following results (dashed histograms) 

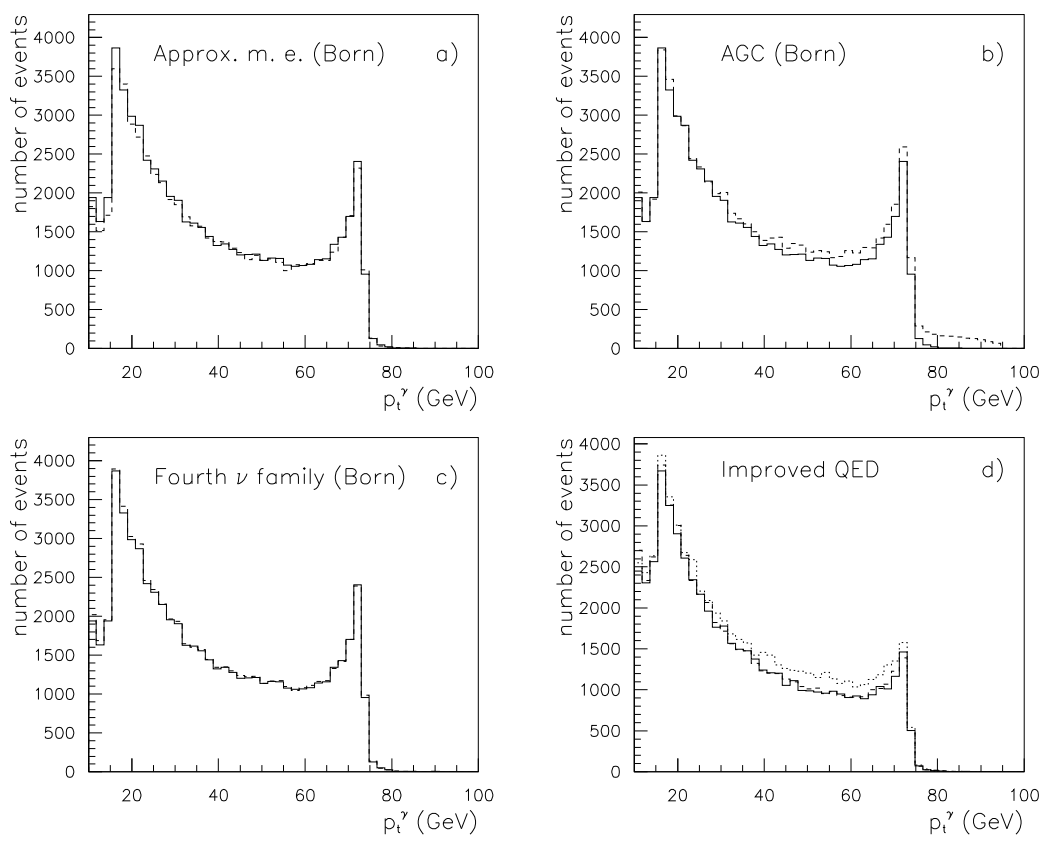

Figure 9: The same as Fig. 8, in the "hard" $p_{t}^{\gamma}$ region.

1. approximate lowest-order photon spectrum of eq. (11), implemented in the released version of NUNUGPV (Fig. 8a, Fig. 9a);

2. exact single-photon matrix element with anomalous $W W \gamma$ coupling, corresponding to the parameters choice $\Delta k_{\gamma}=\lambda_{\gamma}=5$ (Fig. 8b, Fig. 9b);

3. exact single-photon matrix element with additional contribution due to the production of a pair of fourth-generation neutrinos, with standard couplings and mass of $50 \mathrm{GeV}$ (Fig. 8c, Fig. 9c).

As can be seen, an exact treatment of the lowest-order matrix element is mandatory in order to obtain fully reliable exclusive photon distributions and to avoid loss of sensitivity in the search of (small) NP deviations. Also a careful formulation of ISR is necessarily required for an appropriate simulation of photon distributions, as shown in Fig. 8d and Fig. 9d. In these plots the three following different implementations of higher-order QED corrections are compared: improved treatment of ISR via eq. (5) (solid histogram), simulation of ISR via collinear SFs as given by eq. (11) (dashed histogram), treatment of ISR via SFs with $p_{t} / p_{L}$ effects as in the released version of NUNUGPV (dotted histogram). The differences between the three implementations of ISR are certainly comparable or larger than the deviations introduced by NP, clearly illustrating the need of a proper treatment of pre-emission QED effects. In particular, the collinear SFs lead, as expected, 
to an overestimate of events in the soft-photon region, while the implementation of ISR via SFs with $p_{t} / p_{L}$ effects, as in the released version of NUNUGPV, is responsible for an excess of events in the large $p_{t}^{\gamma}$ region, as a consequence of the approximate treatment of $p_{t}$ contributions outside the collinear approximation.
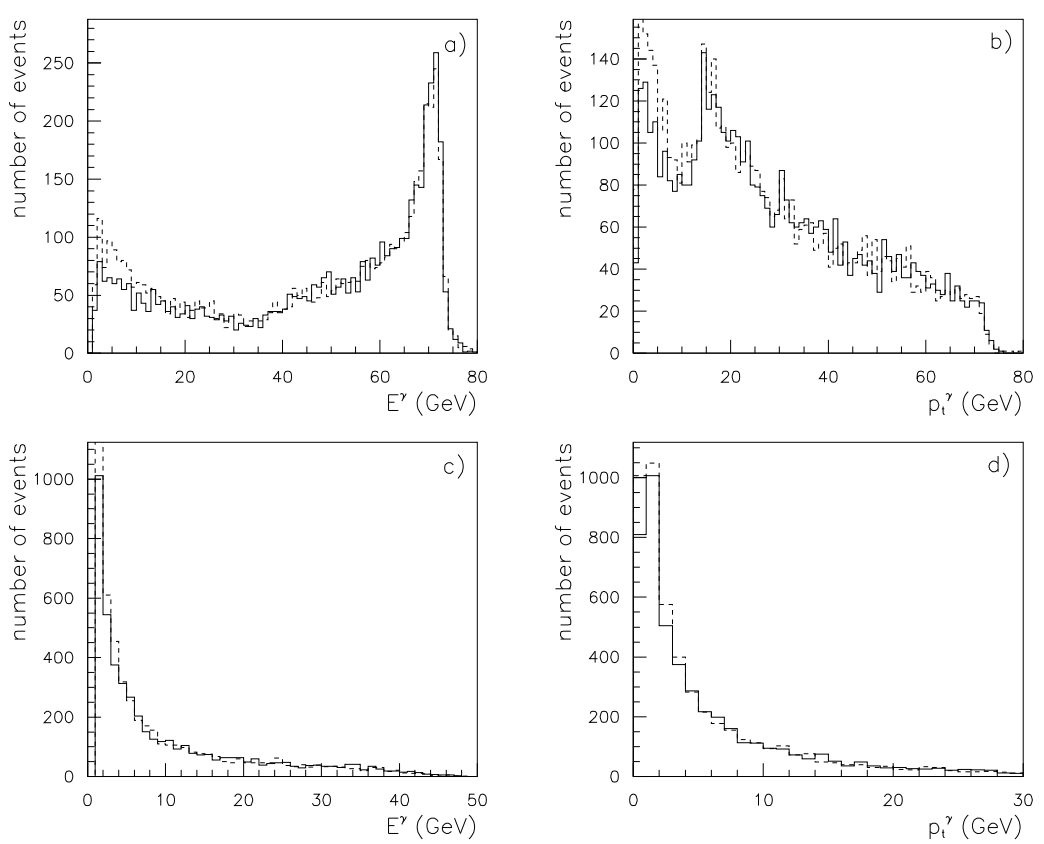

Figure 10: $E_{\gamma}$ and $p_{t}^{\gamma}$ distributions for the leading (Figs. 10a-10b) and next-to-leading photon (Figs. 10c-10d) of the process $e^{+} e^{-} \rightarrow \nu \bar{\nu} \gamma \gamma(\gamma)$ at $\sqrt{s}=190 \mathrm{GeV}$. The cuts on the observed photons are $E_{\gamma}>1 \mathrm{GeV}$ and $12^{\circ}<\vartheta_{\gamma}<168^{\circ}$. ISR correction is implemented according to: eq. (6) (solid line) and eq. (4) (dashed line).

A comparison between photon distributions obtained by means of different simulations of ISR is also shown in Fig. 10 for the $e^{+} e^{-} \rightarrow \nu \bar{\nu} \gamma \gamma(\gamma)$ events. The implementation of ISR via collinear SFs as in eq. (丑) (dotted histograms) is compared with the improved formulation of eq. (6) (solid histograms). The energy and $p_{t}$ distributions of the leading (Figs. 10a-10b) and of the next-to-leading (Figs. 10c-10d) photon are considered. It can be seen that the implementation of ISR via collinear SFs, as done in practice in many computational tools for $\nu \bar{\nu} \gamma \gamma$ final state, lead to an excess of events in the soft-photon region, as already noticed for the $\nu \bar{\nu} \gamma$ signature. Since the soft-photon region is of primary interest for the search of NP effects, a careful treatment of ISR, as given by eqs. (5)-(6), is essential to obtain actually precise predictions for the SM irreducible background. 


\section{Concluding remarks}

The search for new physics in single- and multi-photon final states with large missing energy at LEP and future $e^{+} e^{-}$colliders requires the best knowledge of the SM irreducible background. To this aim, precise calculations (and related computational tools) for the processes $e^{+} e^{-} \rightarrow \nu \bar{\nu} n \gamma$ are presently demanded. Towards such a direction, the tree-level matrix elements for the SM processes with neutrino pairs and up to three photons in the final state have been calculated without any approximation. The exact treatment of the lowest-order transition amplitudes has been seen to be actually necessary in view of an expected precision at the $1 \%$ level. At this accuracy level, also a careful treatment of the (large) effect of the higher-order corrections introduced by ISR is unavoidable. Indeed, it has been shown that the usual implementation of ISR via collinear SFs, which is a good approximation at LEP1 energies and with the usual selection criteria, can lead to an overestimate of the physical cross section much larger than 1\%. In particular, in the presence of a missing mass cut, the ISR overestimate of the integrated cross section reaches the ten percent size. Furthermore, significant effects, whenever compared with typical NP deviations, have been shown to be present also in the photon distributions and should therefore carefully considered in a sensible experimental analysis. The remaining uncertainty in the present study is left to the yet unknown exact $O(\alpha)$ electroweak corrections to the process $e^{+} e^{-} \rightarrow \nu \bar{\nu} \gamma$. Such a complete calculation should be actually desirable to reach a theoretical error not exceeding the $1 \%$ level.

As a result of the present study, an improved version of the event generator NUNUGPV is by now available. It includes the exact SM matrix elements for $\nu \bar{\nu} n \gamma$ production, with $n=1,2,3$, and a careful treatment of the ISR including $p_{t} / p_{L}$ effects. For single-photon production the possibility of studying the effects of anomalous couplings is included. Predictions for the production of a pair of hypothetic massive neutrinos can be also obtained. A sample of numerical results showing the potentials of the new version of NUNUGPV has been shown, with particular emphasis on the impact of the discussed improvements on the integrated cross sections and more exclusive distributions. The program can be used for a full analysis of single- and multi-photon events with missing energy at LEP2 and future high-energy $e^{+} e^{-}$colliders.

\section{Acknowledgements}

We wish to thank J. Busenitz, P. Checchia, C. Matteuzzi, B. Mele and G.W. Wilson for valuable information, useful discussions and interest in our work. 


\section{References}

[1] M. Chemarin, Search for anomalous photon events at LEP, LYCEN-9809, in Proc. of Int. Eur. Conf. on High-Energy Physics, Jerusalem, 19-26 Aug. 1997;

V. Shoutko, Test of QED and the SM with photonic final states at LEP, in Proc. of Int. Eur. Conf. on High-Energy Physics, Jerusalem, 19-26 Aug. 1997.

[2] R. Barate et al., ALEPH Coll., CERN-EP/98-053, Phys. Lett. B 420 (1998) 128;

P. Abreu et al., DELPHI Coll., Phys. Lett. B 380 (1996) 471;

M. Acciarri et al., L3 Coll., Phys. Lett. B 415 (1997) 299;

K. Ackerstaff et al., OPAL Coll., Eur. Phys. J. C2 (1998) 607.

[3] Physics at LEP, J. Ellis and R. Peccei eds., CERN 86-02 (CERN, Geneva, 1986).

[4] Z Physics at LEP1, G. Altarelli, R. Kleiss and C. Verzegnassi eds., CERN 89-08 (CERN, Geneva, 1989).

[5] Physics at LEP2, G. Altarelli, T. Sjöstrand and F. Zwirner eds., CERN 96-01 (CERN, Geneva, 1986).

[6] E. Accomando et al., hep-ph/9705442, Phys. Rep. 299 (1998) 1.

[7] G.F. Giudice, M.L. Mangano, G. Ridolfi, R. Rückl et al., Searches for new physics, in [5], Vol. 1, p. 463;

S. Ambrosanio et al., Nucl. Phys. B 478 (1996) 46, Phys. Rev. D 56 (1997) 1761, Phys. Rev. D 54 (1996) 5395;

J.L. Lopez, D.V. Nanopoulos and A. Zichichi, Phys. Rev. D 55 (1997) 5813, Phys. Rev. Lett. 77 (1996) 5168;

A. Brignole, F. Feruglio and F. Zwirner, Nucl. Phys. B 516 (1998) 13.

[8] A.R. Zerwekh and R. Rosenfeld, Limits on a strong electroweak sector from $e^{+} e^{-} \rightarrow$ $\gamma \gamma+$ Ef at LEP2, IFT-P.027/98, hep-ph/9805329.

[9] G. Montagna, O. Nicrosini and F. Piccinini, Comput. Phys. Commun. 98 (1996) 206.

[10] L. Trentadue et al., Neutrino counting, in [4], Vol. 1, p. 129.

[11] F. Boudjema, B. Mele et al., Standard Model processes, in [5], Vol. 1, p. 207.

[12] K.J.F. Gaemers, R. Gastmans and F.M. Renard, Phys. Rev. D 19 (1979) 1605;

G. Barbiellini, B. Richter and J.L. Siegrist, Phys. Lett. B 106 (1981) 414.

[13] O. Nicrosini and L. Trentadue, Nucl. Phys. B 318 (1989) 1.

[14] G. Montagna, O. Nicrosini, F. Piccinini and L. Trentadue, Nucl. Phys. B 452 (1995) 161.

[15] T. Sjöstrand, Comput. Phys. Commun. 79 (1994) 503, CERN-TH.7112/93. 
[16] S. Jadach, B.F.L. Ward and Z. Wass, Comput. Phys. Commun. 79 (1994) 503.

[17] A. Jachołkowska, J. Kalinowski and Z. Wạs, Higher-order QED corrections to $e^{+} e^{-} \rightarrow \nu \bar{\nu} \gamma$ at LEP2, CERN-TH/98-55, IFT/3/98, hep-ph/9803375.

[18] F.A. Berends et al., Nucl. Phys. B 301 (1988) 583.

[19] R. Miquel, C. Mana and M. Martinez, Z. Phys. C 48 (1990) 309.

[20] M. Böhm and T.H. Sack, Z. Phys. C 35 (1987) 119.

[21] F.A. Berends, G.J.H. Burgers and W.L. van Neerven, Phys. Lett. B 177 (1986) 191; M. Igarashi and N. Nakazawa, Nucl. Phys. B 288 (1987) 301;

H. Veltman, Nucl. Phys. B 312 (1989) 1.

[22] Y. Kurihara, J. Fujimoto, T. Munehisa and Y. Shimizu, Prog. Theor. Phys. 96 (1996) 1223, ibid. 95 (1996) 375;

J. Fujimoto, T. Munehisa and Y. Shimizu, Prog. Theor. Phys. 90 (1993) 177;

K. Kato and T. Munehisa, Phys. Rev. D 39 (1989) 156;

G. Marchesini and B.R. Webber, Nucl. Phys. B 238 (1984) 1;

R. Odorico, Nucl. Phys. B 172 (1980) 157.

[23] E.A. Kuraev and V.S. Fadin, Sov. J. Nucl. Phys. 41 (1985) 466;

G. Altarelli and G. Martinelli, in [3, Vol. 1, p. 47;

O. Nicrosini and L. Trentadue, Phys. Lett. B 196 (1987) 551; Z. Phys. C 39 (1988) 479;

F.A. Berends, G. Burgers and W.L. van Neerven, Nucl. Phys. B 297 (1988) 429.

[24] S. Jadach and B.F.L. Ward, Comput. Phys. Commun. 56 (1990) 351, Phys. Rev. D 38 (1988) 2897;

D.R. Yennie, S. Frautschi and H. Suura, Ann. Phys. (NY) 13 (1961) 379.

[25] O. Nicrosini and L. Trentadue, Phys. Lett. B 231 (1989) 487.

[26] J. Busenitz, Comparison of Single Photon Event Generators at LEP2 Energies, L3 note 2172 (1997), and private communication.

[27] S. Mrenna, Physics backgrounds to supersymmetric signals with two photons and missing mass at LEP, ANL-HEP-PR-97-27, hep-ph/9705441.

[28] S. Ambrosanio et al., Phys. Rev. D 54 (1996) 5395.

[29] T. Ishikawa et al., KEK Report 92-19, 1993;

H. Tanaka, T. Kaneko and Y. Shimizu, Comput. Phys. Commun. 64 (1991) 149;

H. Tanaka, Comput. Phys. Commun. 58 (1990) 153.

[30] E.E. Boos et al., hep-ph/9503280;

E.E. Boos et al., Int. J. Mod. Phys. C5 (1994) 615. 
[31] P. Bain and R. Pain, Acoplanar photons background generators, DELPHI 97-89 CONF 75, HEP'97 \#528, paper submitted to the Int. Eur. Conf. on High-Energy Physics, Jerusalem, 19-26 Aug. 1997.

[32] P. De Causmaecker, R. Gastmans, W. Troost and T.T. Wu, Phys. Lett. B 105 (1981) 215, Nucl. Phys. B 206 (1982) 53;

F.A. Berends et al., Nucl. Phys. B 206 (1982) 61, ibid. 239 (1984) 382, ibid. 239 (1984) 395, ibid. 264 (1986) 243, ibid. 264 (1986) 265;

M. Caffo and E. Remiddi, Helv. Phys. Acta 55 (1982) 339;

G. Passarino, Phys. Rev. D 28 (1983) 2867, Nucl. Phys. B 237 (1984) 249;

F.A. Berends, P.H. Daverveldt and R. Kleiss, Nucl. Phys. B 253 (1985) 442;

R. Kleiss and W.J. Stirling, Nucl. Phys. B 262 (1985) 235, Phys. Lett. B 179 (1986) 159 .

[33] F. Caravaglios and M. Moretti, Phys. Lett. B 358 (1995) 332.

[34] D. Bardin, R. Kleiss et al., Event generators for $W W$ physics, in [5], Vol. 2, p. 3.

[35] M. Moretti, Nucl. Phys. B 484 (1997) 3.

[36] M. Baillargeon, G. Belanger and F. Boudjema, Phys. Lett. B 404 (1997) 124.

[37] F. Caravaglios and M. Moretti, Z. Phys. C 74 (1997) 291.

[38] G. Montagna, M. Moretti, O. Nicrosini and F. Piccinini, Eur. Phys. J. C2 (1998) 483 ;

F. Gangemi, G. Montagna, M. Moretti, O. Nicrosini and F. Piccinini, in $e^{+} e^{-}$Linear Colliders: Physics and Detectors Studies, DESY 97-123E, R. Settles ed., p. 393. 